\title{
NY-ESO-1 (CTAG1B) expression in mesenchymal tumors
}

Makoto Endo ${ }^{1,2,7}$, Marieke A de Graaff ${ }^{3,7}$, Davis R Ingram ${ }^{4}$, Simin Lim¹ ${ }^{1}$, Dina C Lev ${ }^{4}$, Inge H Briaire-de Bruijn ${ }^{3}$, Neeta Somaiah ${ }^{5}$, Judith VMG Bovée ${ }^{3}$, Alexander J Lazar ${ }^{6}$ and Torsten O Nielsen ${ }^{1}$

${ }^{1}$ Department of Pathology and Laboratory Medicine, University of British Columbia, Vancouver, British Columbia, Canada; ${ }^{2}$ Department of Orthopaedic Surgery, Kyushu University, Fukuoka, Japan; ${ }^{3}$ Department of Pathology, Leiden University Medical Center, Leiden, The Netherlands; ${ }^{4}$ Department of Surgical Oncology, The University of Texas MD Anderson Cancer Center, Houston, TX, USA; ${ }^{5}$ Department of Sarcoma Medical Oncology, The University of Texas MD Anderson Cancer Center, Houston, TX, USA and ${ }^{6}$ Department of Pathology, The University of Texas MD Anderson Cancer Center, Houston, TX, USA

\begin{abstract}
New York esophageal squamous cell carcinoma 1 (NY-ESO-1, CTAG1B) is a cancer-testis antigen and currently a focus of several targeted immunotherapeutic strategies. We performed a large-scale immunohistochemical expression study of NY-ESO-1 using tissue microarrays of mesenchymal tumors from three institutions in an international collaboration. A total of 1132 intermediate and malignant and 175 benign mesenchymal lesions were enrolled in this study. Immunohistochemical staining was performed on tissue microarrays using a monoclonal antibody for NY-ESO-1. Among mesenchymal tumors, myxoid liposarcomas showed the highest positivity for NY-ESO-1 (88\%), followed by synovial sarcomas (49\%), myxofibrosarcomas (35\%), and conventional chondrosarcomas (28\%). Positivity of NY-ESO-1 in the remaining mesenchymal tumors was consistently low, and no immunoreactivity was observed in benign mesenchymal lesions. On the basis of these findings, nearly $90 \%$ of myxoid liposarcomas, as well as a significant proportion of synovial sarcomas, myxofibrosarcomas, and conventional chondrosarcomas are good candidates for immunotherapy targeting NY-ESO-1.
\end{abstract}

Modern Pathology (2015) 28, 587-595; doi:10.1038/modpathol.2014.155; published online 21 November 2014

New York esophageal squamous cell carcinoma 1 (NY-ESO-1), encoded by the CTAG1B gene, is a cancer-testis antigen that was identified in 1997 from the serum of a patient with esophageal squamous cell carcinoma. ${ }^{1}$ Cancer-testis antigens such as NY-ESO-1 have attracted increasing attention as immunotherapeutic targets because, among normal tissues, they are expressed only in adult testis germ cells and are atypically re-expressed in many malignancies. $^{2,3} \mathrm{NY}$-ESO-1 is of particular interest to researchers and clinicians because it is highly immunogenic and is expressed in a variety of carcinomas, melanomas, and sarcomas. ${ }^{4} \mathrm{~A}$ recent

Correspondence: Dr TO Nielsen, MD, PhD, Department of Pathology and Laboratory Medicine, University of British Columbia, Room 509-2660 Oak Street, Vancouver, British Columbia V6H 3Z6, Canada.

E-mail: torsten@mail.ubc.ca

${ }^{7}$ These authors contributed equally to this work.

Received 13 June 2014; revised 9 October 2014; accepted 15

October 2014; published online 21 November 2014 clinical trial of adoptive immunotherapy, using genetically modified $\mathrm{T}$ cells directed against NYESO-1, demonstrated objective clinical responses in a number of metastatic synovial sarcoma and melanoma patients with NY-ESO-1-positive tumors. ${ }^{5}$ The results of that clinical trial highlight the potential effectiveness of immunotherapy against tumors expressing NY-ESO-1, and several clinical trials (using antigen sensitization, adoptive T-cell transfer, and dendritic cell vaccine strategies) are currently underway.

Mesenchymal tumors arising from bone or soft tissues comprise many histologic subtypes, which even when taken together occur at a much lower rate than the more common carcinomas, creating a practical barrier to developing new drug therapies. However, the well-defined biology of many sarcomas suggests they may be particularly susceptible to appropriate targeted strategies. Recent studies have reported NY-ESO-1 expression in an especially large proportion of myxoid liposarcomas and synovial sarcomas. $^{2,6-9}$ These studies also revealed the 
absence of NY-ESO-1 expression in their histologic mimics such as myxoma, myxofibrosarcoma, extraskeletal myxoid chondrosarcoma, and lowgrade fibromyxoid sarcoma, and concluded that immunohistochemical staining of NY-ESO-1 is useful not only for candidate selection in immunotherapy, but also for histologic differential diagnosis. A limitation of these previous studies is that the sample sizes were too small to be conclusive and lacked independent external validation.

The goals of this study are to validate published reports of NY-ESO-1 expression in myxoid liposarcoma and synovial sarcoma patients on a larger sample set, and to investigate NY-ESO-1 expression in other mesenchymal tumors not represented in previous studies. To do so, we undertook a multiinstitutional NY-ESO-1 expression survey on tissue microarrays encompassing a wide variety of malignant, intermediate, and benign bone and soft tissue tumors, seeking to determine whether NY-ESO-1 is indeed useful in the differential diagnosis among mesenchymal tumors and to identify subtypes that are most likely to benefit from NY-ESO-1 immunotherapy.

\section{Materials and methods}

\section{Tumor Samples and Tissue Microarrays}

In this study, formalin-fixed paraffin-embedded tissue microarrays from three institutions, the University of British Columbia (UBC), the MD Anderson Cancer Center (MDACC), and the Leiden University Medical Center (LUMC), with a diverse selection of benign, intermediate, and malignant mesenchymal tumors were used. Each tissue microarray contains duplicate, triplicate, or quadruplicate $0.6 \mathrm{~mm}$ (UBC, LUMC), $1.5 \mathrm{~mm}$ (LUMC), or $2.0 \mathrm{~mm}$ (MDACC, LUMC) cores derived from representative viable diagnostic areas identified by a specialized bone and soft tissue tumor pathologist (TON, AJL, JVMGB). Tissue microarrays from UBC included in this study are TMA 01-003, ${ }^{10}$ TMA 03-008, ${ }^{11}$ TMA 06-007, ${ }^{12}$ TMA 06-001B, ${ }^{13}$ TMAs 08-019, 09-006, 10-009, ${ }^{14}$ TMAs $12-004,12-005,12-006,12-010,{ }^{15}$ and TMA MPNST. ${ }^{16}$ From MDACC, myxoid liposarcoma tissue microarrays contained untreated tumors and tumors pre-treated with chemotherapy, radiation, or a combination of both. ${ }^{17,18}$ From the LUMC, tissue microarrays were selected containing chondrosarcomas, ${ }^{19,20}$ osteosarcomas, ${ }^{21}$ myxoid liposarcomas, and a variety of benign and malignant soft tissue tumors. ${ }^{22,23}$

\section{Immunohistochemistry}

Immunohistochemical staining was performed with an autostainer for tissue microarrays of the UBC and MDACC following protocols as described previously. ${ }^{24}$ Briefly, antigen retrieval was performed using the Standard Cell Conditioning 1 (CC1) protocol (Ventana, Tucson, AZ, USA). Sections were incubated with mouse anti-NY-ESO-1 monoclonal antibody (clone E978, Invitrogen, Carlsbad, CA, USA) at 1:25 dilution for $2 \mathrm{~h}$ at room temperature, and then with UltraMap HRP-conjugated anti-mouse IgG secondary antibody for $16 \mathrm{~min}$. The ChromoMap DAB Kit (Ventana) provided the visualization chromogen. Slides were then counterstained with hematoxylin and mounted. Immunohistochemical staining of the tissue microarrays of the LUMC was performed manually; $4 \mu \mathrm{m}$ sections were transferred to adhesive-coated slides (Leica Biosystems, Rijswijk, The Netherlands) and dried overnight at $37^{\circ} \mathrm{C}$. Next slides were deparaffinized in xylene, rehydrated and incubated in $0.3 \%$ methanol $/ \mathrm{H}_{2} \mathrm{O}_{2}$ at room temperature for $20 \mathrm{~min}$ to block the endogenous peroxidase activity. Antigen retrieval was performed by $10 \mathrm{~min}$ microwave treatment in tris/EDTA-solution $(\mathrm{pH}=9.0)$, followed by the addition of the primary NY-ESO-1 / CTAG1B antibody (1:100, clone E978, Invitrogen) and overnight incubation in a moist chamber at room temperature. Next, slides were incubated with PowerVision Poly-HRP (Leica), visualized with $\mathrm{DAB}+$ Substrate Chromogen System (DAKO, Heverlee, Belgium) and counterstained with hematoxylin. All wash steps were carried out in PBS.

In both protocols, the staining was preceded by a titration series on normal testis, and during the actual staining, testis was included as a positive control, and as the tissue present in the negative control reaction (without the addition of the primary antibody).

\section{Digital Images}

Digital images of immunostained and H\&E-stained tissue microarrays of UBC were acquired using a BLISS imager (Bacus Laboratories, Lombard, IL, USA). A relational database was constructed that correlates scoring and identification information with images of each core. This information is publicly accessible at http://www.gpecimage.ubc.ca (username: nyeso1; password: nyeso1).

\section{Evaluation of Immunohistochemistry}

The tissue microarray slides were evaluated under a light microscope or on the scanned images by two to five independent observers (ME, MAG, TON, JVMGB, AJL), including three experienced bone and soft tissue tumor subspecialty pathologists (TON, JVMGB, AJL). NY-ESO-1 immunopositivity was scored in a semiquantitative manner for the intensity $(0=$ negative, $1=$ weak positive, $2=$ moderate positive, $3=$ strong positive) and the percentage of positive tumor cells $(0=0 \%, 1=1-25 \%, 2=26-$ $50 \%, 3=51-75 \%, 4=76-100 \%)$. Each core on the tissue microarrays was scored separately and the 
average score from cores of the same tumor were calculated. Tumor samples with more than 50\% of positive tumor cells and a staining intensity of moderate or strong (2 or 3 ) were defined as 'positive'. This cutoff value was chosen to be consistent with criteria used in an ongoing clinical trial of NY-ESO-1 immunotherapy. ${ }^{5}$ In addition, cross comparison of manual and autostaining revealed the manual method generated a weak background stain not seen with the automated platform. However, the scoring system, which considered weak (intensity 1) staining as negative meant that the cases defined as 'positive' were consistently categorized and equally discernible with both staining methods. Comparison of TMA with the whole section was performed on a comparison set of 11 cases, and the staining results were found to be concordant.

\section{Statistical Analysis}

A chi-square test was used to assess the differences between histological subtypes; statistical significance was defined as $P<0.05$. Data analysis was performed with JMP software (version 9.0.2, JMP Software, Cary, NC, USA).

\section{Results}

In total, specimens from 1132 malignant and intermediate bone and soft tissue tumors, 175 benign bone and soft tissue tumors, 50 non-mesenchymal tumors, 6 non-tumorous lesions, and 8 samples of normal fat were available for evaluation (Tables 1-3). There were 38 subtypes of malignant and intermediate tumors and 21 different benign mesenchymal lesions in this cohort. The subtypes with more than 50 samples available included myxoid liposarcoma ( $n=201$ in total; 158 untreated cases, and 43 tumors that received treatment prior to resection), gastrointestinal stromal tumor $(n=146)$, conventional chondrosarcoma $(n=142)$ malignant peripheral nerve sheath tumor $(n=82)$, osteosarcoma $(n=80)$, synovial sarcoma $(n=69)$, endometrial stromal sarcoma $(n=58)$, and leiomyosarcoma $(n=55)$.

Among a wide variety of mesenchymal tumors, myxoid liposarcomas that had not received neoadjuvant treatment prior to resection showed the highest positivity for NY-ESO-1 (CTAG1B): $88.0 \%$ (139/158). The high number of positive cases was observed both in the autostained cohort cases from UBC and MDACC (85.9\%; 110/128) and in the manually stained cohort cases from LUMC $(96.7 \%$; 29/30). Even among myxoid liposarcomas that received chemotherapy or radiation prior to resection, NY-ESO-1 expression was still detectable in $41.9 \%(18 / 43)$ of cases, although this was significantly lower $(P<0.0001)$ compared with the cases without preoperative treatment. Expression was seen both in myxoid as well as round cell areas. In an additional $37.2 \%(16 / 43)$ of these neoadjuvantly treated tumors, weak staining in a small subset of the tumor cells was present; the remaining $20.9 \%$ (9/43) did not reveal any residual immunoreactivity. In comparison, the other liposarcomas-including well-differentiated (4.8\% positivity; $1 / 21)$ and dedifferentiated $(14.3 \% ; 1 / 7)$ subtypes-showed NYESO-1 expression in only a minor subset of the cases; none of the tested pleomorphic liposarcomas revealed NY-ESO-1 expression (0\%; 0/11). NY-ESO1 positivity in myxoid liposarcomas was significantly higher than in the other liposarcomas $(P<0.0001)$ or in the other malignant and intermediate bone and soft tissue tumors $(P<0.0001)$.

NY-ESO-1 expression was observed in almost half $(49.3 \% ; 34 / 69)$ of the synovial sarcomas. In addition, positivity was found in conventional chondrosarcoma $(28.2 \% ; 40 / 142)$ and dedifferentiated chondrosarcoma $(10.0 \% ; 3 / 30)$. In the conventional chondrosarcomas, there was no correlation of NY-ESO-1 expression and histological grade. Two of the positive dedifferentiated chondrosarcomas revealed NY-ESO-1 expression only in the dedifferentiated component and the third positive case showed immunoreactivity in the well-differentiated component.

Other sarcomas with a substantial percentage of positive tumors include myxofibrosarcoma $(35.3 \%$; 6/17), leiomyosarcoma $(14.5 \% ; 8 / 55)$, dermatofibrosarcoma protuberans $(11.1 \% ; 2 / 18$-including one of nine cases with fibrosarcomatous change), and undifferentiated pleomorphic sarcoma (11.1\%; 3/27). Pleomorphic rhabdomyosarcoma (33.3\%; $1 / 3)$ and fibrosarcoma $(12.5 \% ; 1 / 8)$ also presented positivity in a minority of the cases, although the sample size was small. The 175 benign bone and soft tissue tumors, the 6 non-tumorous lesions, and the 8 normal fat samples were all negative for NY-ESO-1. In the group of the non-mesenchymal tumors, $10 \%$ (1/10) of the desmoplastic melanomas and none of the other melanomas showed NY-ESO-1 positivity.

Positive controls of formalin-fixed, paraffinembedded testis sections revealed strong NY-ESO-1 expression among spermatogonia and spermatocytes in the seminiferous tubules. No immunoreactivity was observed in any of the negative controls. Representative results of a selection of malignant bone and soft tissue tumors are shown in Figures 1-3.

\section{Discussion}

To the best of our knowledge, this is the largest-scale NY-ESO-1 (CTAG1B) expression study in bone and soft tissue tumors, including over 1300 specimens representing 38 malignant and borderline and 21 benign histologic subtypes. Of special note is the inclusion in our study of 201 myxoid liposarcomas and 69 synovial sarcomas-two sarcoma subtypes 
Table 1 Immunohistochemical NY-ESO-1 (CTAG1B) expression in intermediate and malignant bone and soft tissue tumors

\begin{tabular}{|c|c|c|c|c|c|c|c|c|c|}
\hline \multicolumn{4}{|l|}{ TMAs UBC and MDACC } & \multicolumn{3}{|c|}{ TMAs LUMC } & \multicolumn{3}{|c|}{ Total } \\
\hline $\begin{array}{l}\text { Maligant and intermediate bone and } \\
\text { soft tissue tumors }\end{array}$ & Cases & $\begin{array}{c}\mathrm{N} \\
\text { positive }\end{array}$ & $\begin{array}{l}\% \text { Pos } \\
\text { cases }\end{array}$ & Cases & $\begin{array}{c}\mathrm{N} \\
\text { positive }\end{array}$ & $\begin{array}{l}\% \text { Pos } \\
\text { cases }\end{array}$ & Cases & $\begin{array}{c}\mathrm{N} \\
\text { positive }\end{array}$ & $\begin{array}{l}\% \text { Pos } \\
\text { cases }\end{array}$ \\
\hline \multicolumn{10}{|l|}{ Soft tissue } \\
\hline \multicolumn{10}{|l|}{ Adipocytic tumors } \\
\hline Liposarcoma, dedifferentiated & 5 & 0 & 0.0 & 2 & 1 & 50.0 & 7 & 1 & 14.3 \\
\hline Liposarcoma, myxoid & 128 & 110 & 85.9 & 30 & 29 & 96.7 & 158 & 139 & $\mathbf{8 8 . 0}$ \\
\hline Liposarcoma, myxoid, post-treatment & 43 & 18 & 41.9 & & & & 43 & 18 & 41.9 \\
\hline Liposarcoma, pleomorphic & 7 & 0 & 0.0 & 4 & 0 & 0.0 & 11 & 0 & 0.0 \\
\hline Liposarcoma, well-differentiated & 13 & 1 & 7.7 & 8 & 0 & 0.0 & 21 & 1 & 4.8 \\
\hline \multicolumn{10}{|l|}{ Fibroblastic/myofibroblastic tumors } \\
\hline Desmoid-type fibromatosis & 8 & 0 & 0.0 & & & & 8 & 0 & 0.0 \\
\hline Dermatofibrosarcoma protuberans & 17 & 2 & 11.8 & 1 & 0 & 0.0 & 18 & 2 & 11.1 \\
\hline Solitary fibrous tumor & 15 & 0 & 0.0 & 2 & 0 & 0.0 & 17 & 0 & 0.0 \\
\hline Fibrosarcoma & 8 & 1 & 12.5 & & & & 8 & 1 & 12.5 \\
\hline Myofibroblastic sarcoma & & & & 2 & 0 & 0.0 & 2 & 0 & 0.0 \\
\hline Myxofibrosarcoma & & & & 17 & 6 & 35.3 & 17 & 6 & 35.3 \\
\hline Low-grade fibromyxoid sarcoma & 5 & 0 & 0.0 & & & & 5 & 0 & 0.0 \\
\hline Sclerosing epithelioid fibrosarcoma & 1 & 0 & 0.0 & & & & 1 & 0 & 0.0 \\
\hline \multicolumn{10}{|l|}{ Smooth muscle tumors } \\
\hline Leiomyosarcoma & 5 & 0 & 0.0 & 50 & 8 & 16.0 & 55 & 8 & 14.5 \\
\hline \multicolumn{10}{|l|}{ Skeletal muscle tumors } \\
\hline Rhabdomyosarcoma, alveolar & 9 & 0 & 0.0 & & & & 9 & 0 & 0.0 \\
\hline Rhabdomyosarcoma, embryonal & 12 & 0 & 0.0 & 2 & 1 & 50.0 & 14 & 1 & 7.1 \\
\hline Rhabdomyosarcoma, pleomorphic & & & & 3 & 1 & 33.3 & 3 & 1 & 33.3 \\
\hline \multicolumn{10}{|l|}{ Vascular tumors of soft tissue } \\
\hline Angiosarcoma & & & & 1 & 0 & 0.0 & 1 & 0 & 0.0 \\
\hline Epithelioid angiosarcoma & 4 & 0 & 0.0 & & & & 4 & 0 & 0.0 \\
\hline Epithelioid hemangioendothelioma & 1 & 0 & 0.0 & & & & 1 & 0 & 0.0 \\
\hline \multicolumn{10}{|l|}{ Gastrointestinal stromal tumors } \\
\hline Gastrointestinal stromal tumor & 143 & 0 & 0.0 & 3 & 0 & 0.0 & 146 & 0 & 0.0 \\
\hline \multicolumn{10}{|l|}{ Nerve sheath tumors } \\
\hline Malignant peripheral nerve sheath tumor & 75 & 2 & 2.7 & 7 & 0 & 0.0 & 82 & 2 & 2.4 \\
\hline \multicolumn{10}{|l|}{ Tumors of uncertain differentiation } \\
\hline Alveolar soft part sarcoma & 8 & 0 & 0.0 & & & & 8 & 0 & 0.0 \\
\hline Angiomatoid fibrous histiocytoma & 2 & 0 & 0.0 & & & & 2 & 0 & 0.0 \\
\hline Chondrosarcoma, extraskeletal myxoid & 5 & 0 & 0.0 & & & & 5 & 0 & 0.0 \\
\hline Clear cell sarcoma & 8 & 0 & 0.0 & & & & 8 & 0 & 0.0 \\
\hline Desmoplastic small round cell tumor & 2 & 0 & 0.0 & & & & 2 & 0 & 0.0 \\
\hline Epithelioid sarcoma & 7 & 0 & 0.0 & & & & 7 & 0 & 0.0 \\
\hline Malignant myoepithelioma & 1 & 0 & 0.0 & & & & 1 & 0 & 0.0 \\
\hline Synovial sarcoma & 63 & 30 & 47.6 & 6 & 4 & 66.7 & 69 & 34 & 49.3 \\
\hline \multicolumn{10}{|l|}{ Undifferentiated/unclassified sarcomas } \\
\hline Undifferentiated pleomorphic sarcoma & 5 & 1 & 20.0 & 22 & 2 & 9.1 & 27 & 3 & 11.1 \\
\hline Undifferentiated spindle cell sarcoma & & & & 5 & 0 & 0.0 & 5 & 0 & 0.0 \\
\hline \multicolumn{10}{|l|}{ Endometrial stromal sarcoma } \\
\hline Endometrial stromal sarcoma & 58 & 3 & 5.2 & & & & 58 & 3 & 5.2 \\
\hline \multicolumn{10}{|l|}{ Bone } \\
\hline \multicolumn{10}{|l|}{ Chondrogenic tumors } \\
\hline Chondrosarcoma, conventional & 29 & 2 & 6.9 & 113 & 38 & 33.6 & 142 & 40 & 28.2 \\
\hline Chondrosarcoma, clear cell & & & & 18 & 0 & 0.0 & 18 & 0 & 0.0 \\
\hline Chondrosarcoma, dedifferentiated & 4 & 0 & 0.0 & 26 & 3 & 11.5 & 30 & 3 & 10.0 \\
\hline Chondrosarcoma, mesenchymal & 3 & 0 & 0.0 & 15 & 0 & 0.0 & 18 & 0 & 0.0 \\
\hline Osteogenic tumors & & & & & & & & & \\
\hline Osteosarcoma & 4 & 1 & 25.0 & 76 & 4 & 5.3 & 80 & 5 & 6.3 \\
\hline Miscellaneous tumors & & & & & & & & & \\
\hline Ewing sarcoma & 21 & 0 & 0.0 & & & & 21 & 0 & 0.0 \\
\hline
\end{tabular}

Bold indicates entities with a significant fraction of positive cases. 
Table 2 Immunohistochemical NY-ESO-1 expression in benign bone and soft tissue tumors

\begin{tabular}{|c|c|c|c|c|c|c|c|c|c|}
\hline \multicolumn{4}{|l|}{ TMAs UBC and MDACC } & \multicolumn{3}{|c|}{ TMAs LUMC } & \multicolumn{3}{|c|}{ Total } \\
\hline $\begin{array}{l}\text { Benign bone and soft } \\
\text { tissue tumors }\end{array}$ & Cases & $\begin{array}{c}\mathrm{N} \\
\text { positive }\end{array}$ & $\begin{array}{l}\% \text { Pos } \\
\text { cases }\end{array}$ & $\begin{array}{l}\text { Scorable } \\
\text { cases }\end{array}$ & $\begin{array}{c}\mathrm{N} \\
\text { positive }\end{array}$ & $\begin{array}{l}\% \text { Pos } \\
\text { cases }\end{array}$ & $\begin{array}{l}\text { Scorable } \\
\text { cases }\end{array}$ & $\begin{array}{c}\mathrm{N} \\
\text { positive }\end{array}$ & $\begin{array}{l}\% \text { Pos } \\
\text { cases }\end{array}$ \\
\hline \multicolumn{10}{|l|}{ Soft tissue } \\
\hline Atypical fibroxanthoma & 2 & 0 & 0.0 & & & & 2 & 0 & 0.0 \\
\hline Congenital mesoblastic nephroma & 2 & 0 & 0.0 & & & & 2 & 0 & 0.0 \\
\hline Fetal rhabdomyoma & 2 & 0 & 0.0 & & & & 2 & 0 & 0.0 \\
\hline Fibroma/fibromatosis & 36 & 0 & 0.0 & 4 & 0 & 0.0 & 40 & 0 & 0.0 \\
\hline Granuloma annulare & 1 & 0 & 0.0 & & & & 1 & 0 & 0.0 \\
\hline Leiomyoma & & & & 6 & 0 & 0.0 & 6 & 0 & 0.0 \\
\hline Lipoma & 7 & 0 & 0.0 & 7 & 0 & 0.0 & 14 & 0 & 0.0 \\
\hline Lipoma, spindle cell & 10 & 0 & 0.0 & & & & 10 & 0 & 0.0 \\
\hline Myofibroma and myofibromatosis & 6 & 0 & 0.0 & & & & 6 & 0 & 0.0 \\
\hline Myositis ossificans & 2 & 0 & 0.0 & & & & 2 & 0 & 0.0 \\
\hline Myxoma & & & & 3 & 0 & 0.0 & 3 & 0 & 0.0 \\
\hline Neurofibroma & 33 & 0 & 0.0 & & & & 33 & 0 & 0.0 \\
\hline Nodular fasciitis & 1 & 0 & 0.0 & & & & 1 & 0 & 0.0 \\
\hline Perineurioma & 3 & 0 & 0.0 & & & & 3 & 0 & 0.0 \\
\hline Schwannoma & 21 & 0 & 0.0 & 3 & 0 & 0.0 & 24 & 0 & 0.0 \\
\hline Tenosynovial giant cell tumor & & & & 1 & 0 & 0.0 & 1 & 0 & 0.0 \\
\hline \multicolumn{10}{|l|}{ Bone } \\
\hline Chondroblastoma & 4 & 0 & 0.0 & & & & 4 & 0 & 0.0 \\
\hline Chondromyxoid fibroma & 3 & 0 & 0.0 & & & & 3 & 0 & 0.0 \\
\hline Enchondroma & 6 & 0 & 0.0 & 5 & 0 & 0.0 & 11 & 0 & 0.0 \\
\hline Osteochondroma & & & & 6 & 0 & 0.0 & 6 & 0 & 0.0 \\
\hline Synovial chondromatosis & & & & 1 & 0 & 0.0 & 1 & 0 & 0.0 \\
\hline
\end{tabular}

Table 3 Immunohistochemical NY-ESO-1 expression in non-mesenchymal tumors and in several non-tumorous lesions

\begin{tabular}{|c|c|c|c|c|c|c|c|c|c|}
\hline & \multicolumn{3}{|c|}{ TMAs UBC and MDACC } & \multicolumn{3}{|c|}{ TMAs LUMC } & \multicolumn{3}{|c|}{ Total } \\
\hline & Cases & $\begin{array}{c}\mathrm{N} \\
\text { positive }\end{array}$ & $\begin{array}{l}\% \text { Pos } \\
\text { cases }\end{array}$ & $\begin{array}{c}\text { Scorable } \\
\text { cases }\end{array}$ & $\begin{array}{c}\mathrm{N} \\
\text { positive }\end{array}$ & $\begin{array}{l}\% \text { Pos } \\
\text { cases }\end{array}$ & $\begin{array}{c}\text { Scorable } \\
\text { cases }\end{array}$ & $\begin{array}{c}\mathrm{N} \\
\text { positive }\end{array}$ & $\begin{array}{l}\% \text { Pos } \\
\text { cases }\end{array}$ \\
\hline \multicolumn{10}{|l|}{ Non-mesenchymal tumors } \\
\hline Hepatoblastoma & 2 & 0 & 0.0 & & & & 2 & 0 & 0.0 \\
\hline Melanoma & 7 & 0 & 0.0 & & & & 7 & 0 & 0.0 \\
\hline Melanoma, desmoplastic & 10 & 1 & 10.0 & & & & 10 & 1 & 10.0 \\
\hline Squamous cell carcinoma & 8 & 0 & 0.0 & & & & 8 & 0 & 0.0 \\
\hline Wilms tumor & 23 & 0 & 0.0 & & & & 23 & 0 & 0.0 \\
\hline \multicolumn{10}{|l|}{ Non-tumorous lesions } \\
\hline Granuloma annulare & 1 & 0 & 0.0 & & & & 1 & 0 & 0.0 \\
\hline Rheumatoid nodule & 5 & 0 & 0.0 & & & & 5 & 0 & 0.0 \\
\hline Normal fat & 8 & 0 & 0.0 & & & & 8 & 0 & 0.0 \\
\hline
\end{tabular}

that have been considered to be potentially good candidates for NY-ESO-1 immunotherapy. We employed a cutoff value for immunohistochemical positivity by reference to the eligibility of an antecedent clinical trial of immunotherapy targeting NY-ESO- $1 .{ }^{5}$

Myxoid liposarcoma represents $20-30 \%$ of all liposarcomas, mostly occurs in young adults, and is characterized by a translocation $\mathrm{t}(12 ; 16)(\mathrm{q} 13 ; \mathrm{p} 11)$ or, in a few percent of the cases, a $\mathrm{t}(12 ; 22)(\mathrm{q} 13 ; \mathrm{q12})$, leading to the chimeric fusion product FUS-DDIT3 or EWSR1-DDIT3, respectively. ${ }^{25,26}$ According to the literature, one-third of myxoid liposarcoma patients develop distant metastases, ${ }^{18,27,28}$ so effective systemic therapy is needed to improve the prognosis of many patients. Previous studies reported NY-ESO-1 expression in $95-100 \%$ of myxoid liposarcomas; these results were based on sample sizes ranging from 25 to 38 cases. ${ }^{6,9}$ Our larger study revealed that 139 out of the $158(88.0 \%)$ untreated myxoid liposarcomas were positive for NY-ESO-1, and concurs with the rate of positivity reported in the previous studies. The slightly lower rate of positivity might be attributable to the fact that some tumors only display a focal NY-ESO-1 positivity, which might be missed because of the use of tissue microarrays. Hemminger et $a l^{9}$ reported that other liposarcoma subtypes, lipomas, and other myxoid mesenchymal tumors were negative for NY-ESO-1, suggesting that NY-ESO-1 could be a 

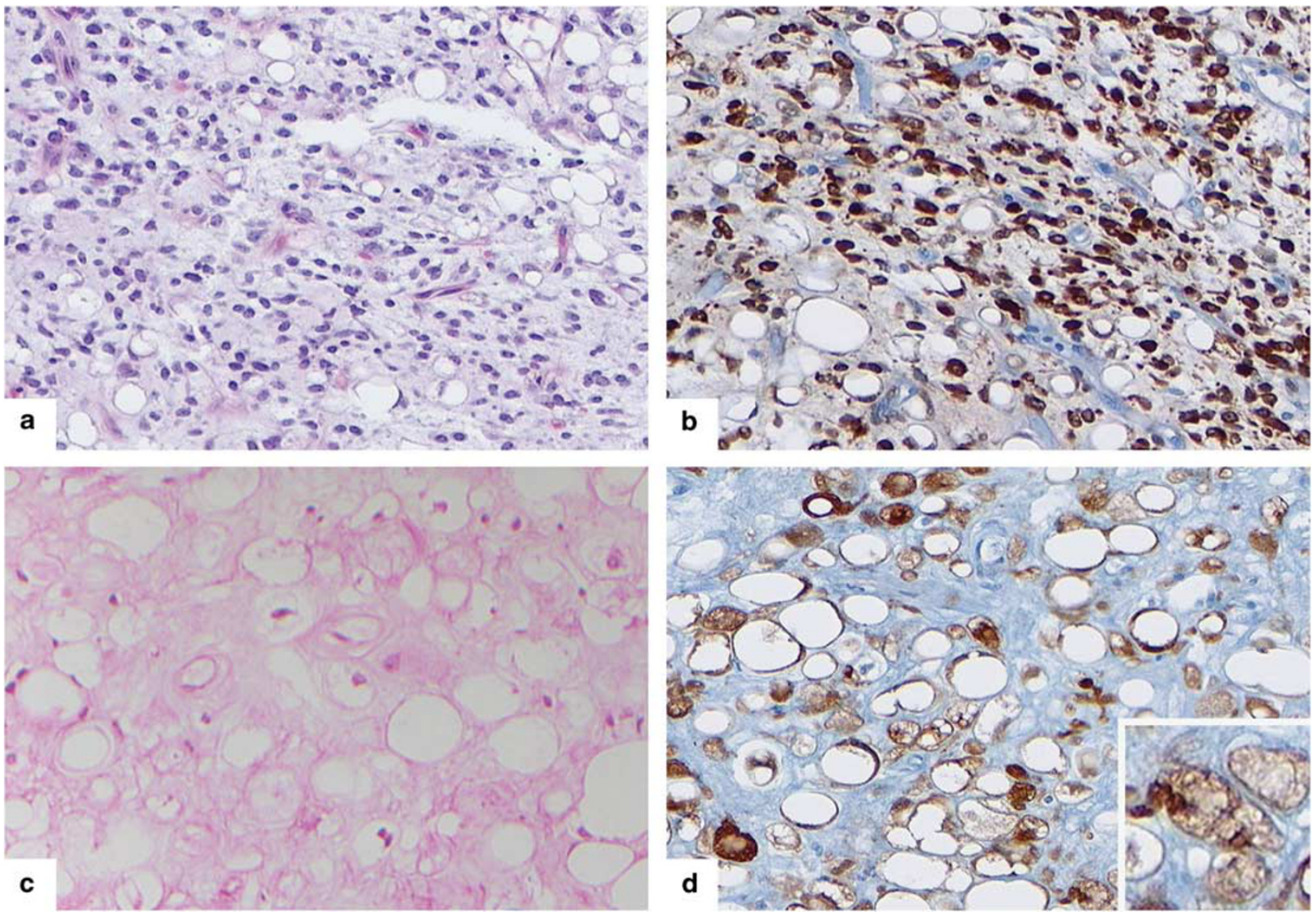

Figure 1 Immunohistochemical staining of NY-ESO-1 (CTAG1B) in myxoid and round cell liposarcomas. Myxoid liposarcoma without treatment prior to resection revealed strong nuclear immunoreactivity in the majority of the tumor cells: (a) H\&E, (b) NY-ESO-1 immunohistochemistry. Myxoid liposarcoma after neoadjuvant treatment (with both radiation and chemotherapy) showed typical histologic changes of hyalinization (c) but retained nuclear and cytoplasmic staining (d).

sensitive and specific immunohistochemical marker to differentiate myxoid liposarcoma from tumors with overlapping histomorphological features. In this study, several mesenchymal tumors with myxoid or lipogenic differentiation were evaluated, including well-differentiated liposarcoma (4.8\%; $1 / 21)$, dedifferentiated liposarcoma $(14.3 \% ; 1 / 7)$, spindle cell lipoma $(0 \% ; 0 / 10)$, lipoma $(0 \% ; 0 / 14)$, normal fat tissue $(0 \% ; 0 / 8)$, myxoma $(0 \% ; 0 / 3)$, and myxofibrosarcoma $(35.3 \% ; 6 / 17)$. Comparing those mimics, myxoid liposarcoma shows remarkably strong immunoreactivity for NY-ESO-1 (88.0\%) and therefore its expression helps to discriminate myxoid liposarcoma from tumors in the differential diagnosis.

Interestingly, chemotherapy- or radiation-pretreated myxoid liposarcomas showed a lower positivity for NY-ESO-1. The biological mechanism behind the decreasing NY-ESO-1 positivity after the preoperative treatment is unknown; however, some of these samples at histological examination showed a low tumor cell density with hyalinization, reactive matrix, and weak NY-ESO-1 staining below the intensity required to label the tumor as positive. NY-
ESO-1 expression is still more frequent even in treated myxoid liposarcomas than in other sarcomas; therefore, NY-ESO-1-targeted immunotherapy combined with established therapies remains an attractive potential strategy. This observed reduction in NYESO-1 positivity in post-treatment myxoid liposarcoma was not seen in a set of eight additional synovial sarcoma specimens, in which all retained strongly positive expression following neoadjuvant treatment (radiation $+/$ - chemotherapy) (Data not shown).

Synovial sarcoma is a translocation-associated sarcoma characterized by the fusion gene, SS18$S S X 1,-S S X 2$, or $-S S X 4 .^{29}$ More than half of the patients are teenagers and young adults. The development of novel systemic therapies is important because metastasis of synovial sarcoma is common (overall 5-year metastasis-free survival rate $51.1 \%)^{30}$ Jungbluth et $a l^{31}$ first investigated NY-ESO-1 expression in synovial sarcoma and found that $80 \%(20 / 25)$ of the samples expressed NY-ESO-1. Recently, Lai et $a l^{7}$ investigated 50 synovial sarcomas and reported that $76 \%(38 / 50)$ of synovial sarcomas expressed NY-ESO-1 in a strong and diffuse pattern. Our study revealed 

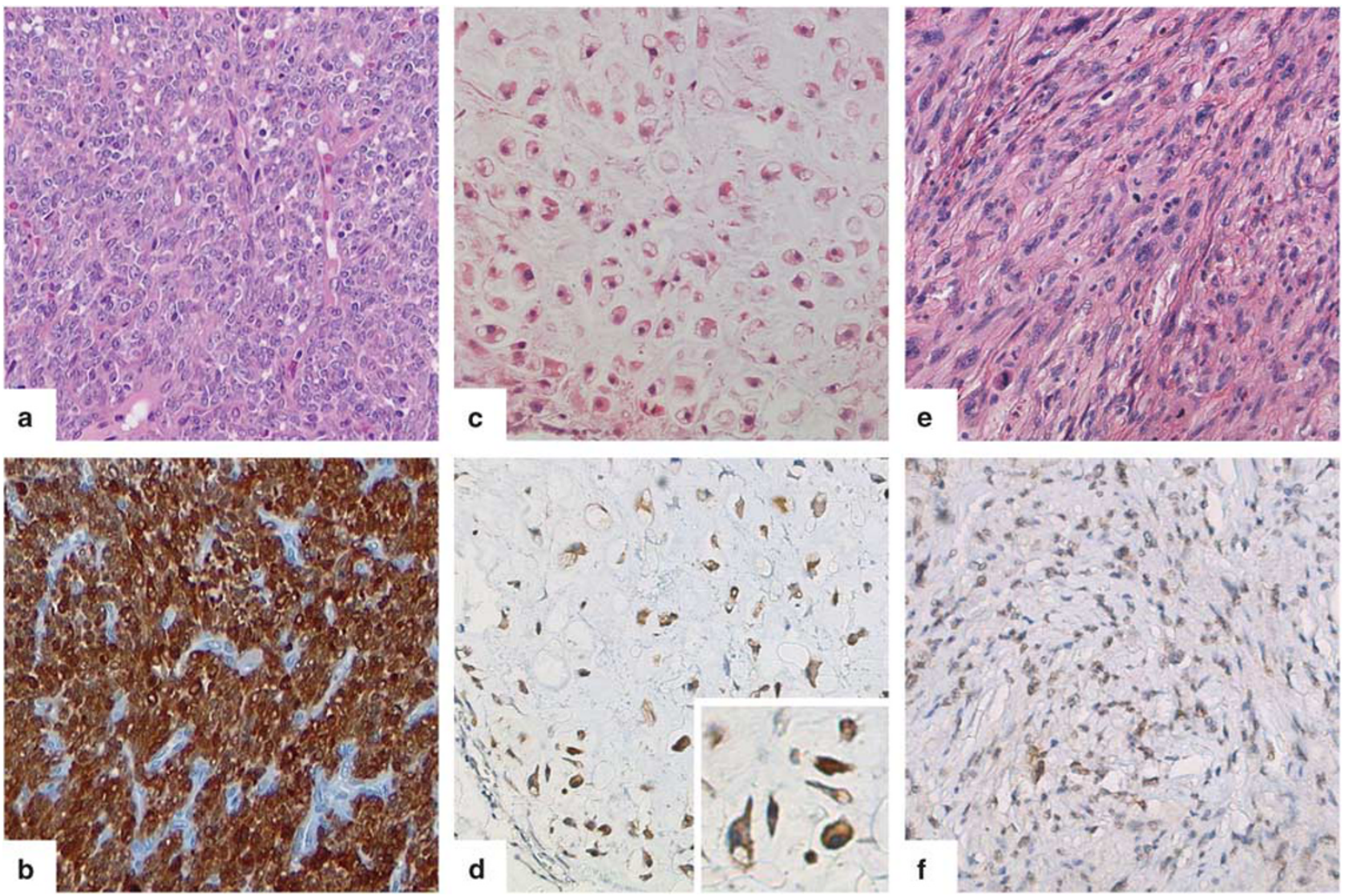

Figure 2 Other sarcomas with positive immunohistochemical staining for NY-ESO-1. (a, b): Synovial sarcoma with strong staining of all tumor cells. (c, d), incl. inset: Conventional chondrosarcoma with prominent cytoplasmic staining. (e, f): Myxofibrosarcoma with moderate immunoreactivity.

$49.3 \%$ positivity across 69 cases of synovial sarcoma, somewhat lower than that of the previous papers, possibly because of the decreased sensitivity using the tissue microarray platform. Considering the combined results from our and previous studies, approximately half to three quarters of synovial sarcomas show NY-ESO-1 expression.

In a recent clinical trial using genetically engineered autologous $\mathrm{T}$ lymphocytes reactive with NY-ESO-1, four of the six advanced synovial sarcoma patients who received immunotherapy showed documented partial responses; ${ }^{5}$ worth noting is that this response lasted at least 18 months in one patient. This result has encouraged the development of NY-ESO-1-targeted immunotherapy not only for synovial sarcomas, but also for other NY-ESO-1 expressing sarcomas including myxoid liposarcomas. Public information on clinicaltrials.gov lists at least 14 clinical trials employing vaccine, autologous T-cell, or dendritic cell transduction immunotherapy strategies that test patients with NY-ESO-1-expressing sarcomas, mostly in active recruitment phases in 2014. Some vaccine studies use a low NY-ESO-1 positivity cutoff value of $>5 \%$, hoping that the vaccine can still elicit an immune response in these patients.
Primary central chondrosarcoma is a bone sarcoma, accounting for approximately $20 \%$ of malignant bone tumors. ${ }^{28}$ As it is highly resistant to existing chemotherapeutic agents, the search for new targeted therapies is ongoing. Our study revealed positivity for NY-ESO-1 in $28.2 \% \quad(40 / 142)$ of conventional chondrosarcoma and $10.0 \%$ (3/30) of dedifferentiated chondrosarcoma specimens, whereas clear cell and mesenchymal chondrosarcomas were all negative. Lai et $a l^{7,32}$ reported that one out of two tested chondrosarcomas showed immunoreactivity for NY-ESO-1, and a relatively low NY-ESO-1 mRNA expression level was found in 4 out of 11 cases in another study. Although the number of positive cases is relatively low, immunotherapy may be of significance in a subset of these patients, who are not included in the eligibility criteria for some of the existing clinical trials.

Apart from the 6 of 17 tested myxofibrosarcomas and 1 of 3 pleomorphic rhabdomyosarcomas, the other tested types of mesenchymal tumors were almost uniformly negative for NY-ESO-1 expression. Although our study included over 1132 cases, the numbers of some rare sarcomas are too limited to draw final conclusions on NY-ESO-1 expression in these subtypes. 

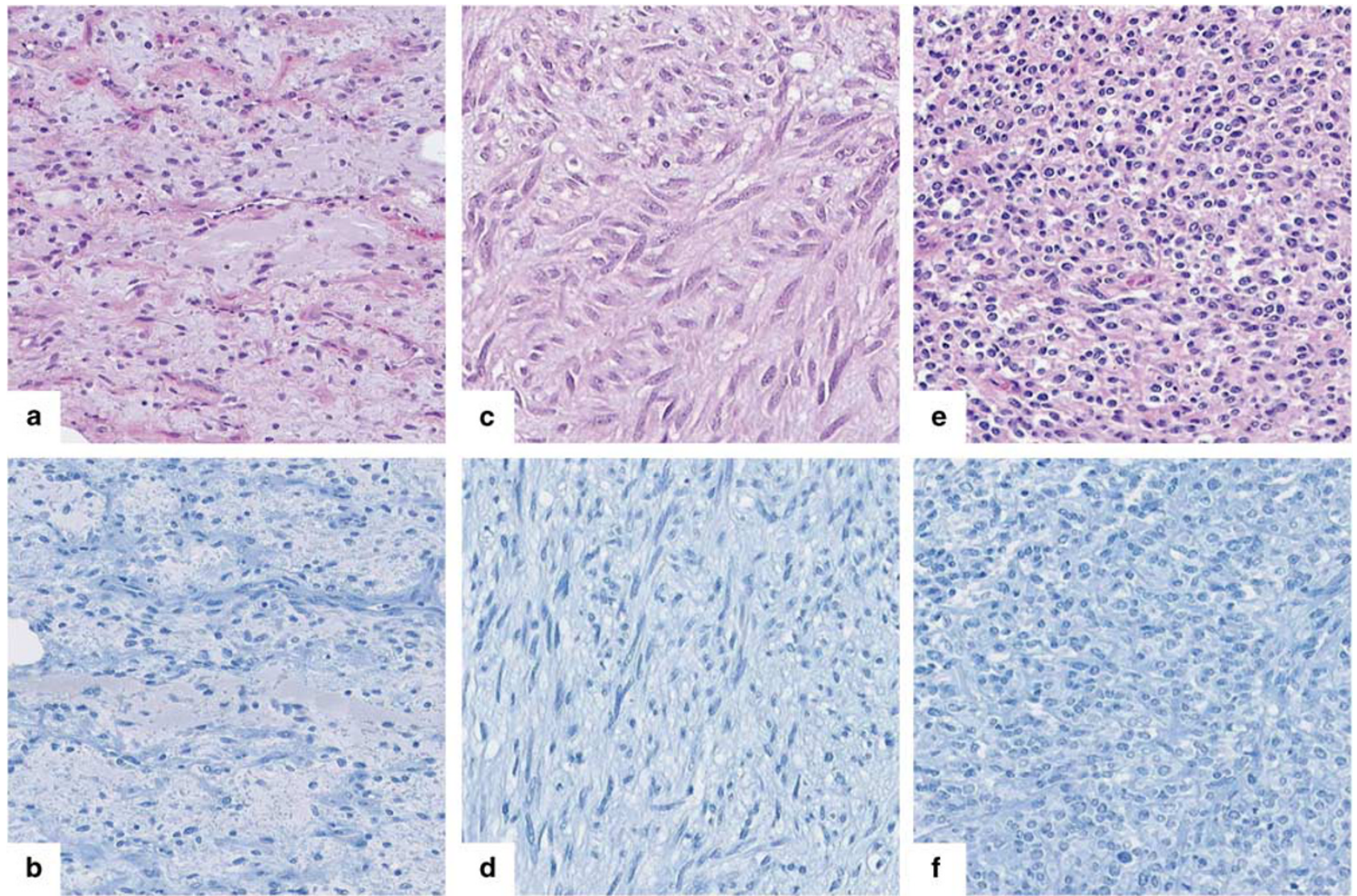

Figure 3 Soft tissue sarcomas without NY-ESO-1 immunoreactivity. (a, b): Myxoid liposarcoma. (c, d): Gastrointestinal stromal tumor. (e, f): Malignant peripheral nerve sheath tumor.

In summary, we confirm that NY-ESO-1 can be reliably detected by immunohistochemistry. Using previously described scoring criteria, but based on much larger numbers of samples than have been assessed previously, we confirm very high frequencies of NY-ESO-1 expression in myxoid liposarcomas, high frequencies in synovial sarcomas, and we find similar levels of expression in $28.2 \%$ of conventional chondrosarcomas. Clinical trials of NY-ESO-1 targeted therapies should be open to patients with positive histologies, and NY-ESO-1 expression by immunohistochemistry should be considered as a potential entry criteria or integrated stratification as a potential predictive marker for such therapeutic strategies. NY-ESO-1 positivity in the remaining subtypes of mesenchymal tumors was rare. Benign bone and soft tissue lesions showed no positivity for NY-ESO-1, supporting the limited level of expression of this biomarker in normal tissues and the possibility that targeted therapies will have limited side effects.

\section{Acknowledgments}

We thank Christine Chow for her excellent technical assistance. We thank Anne-Marie Cleton-Jansen,
Dorien van der Geest, Marieke Kuijjer, Danielle Meijer, Jolieke van Oosterwijk, and Heidi van Paassen for the help with the construction of the LUMC tissue micro arrays and the collection of the clinical data. We would like to thank EuroBoNeT partners Nick Athanasou, the University of Oxford, UK; Soren Daugaard, Department of Pathology, RH, Denmark, Bernadette Liegl, Department of Pathology, Medizinische Universität Graz, Austria and Pierro Picci, Department of Pathology, Rizzoli Institute, Bologna, Italy for providing cases of rare chondrosarcoma subtypes. We also thank Samuel Leung for building the online relational database. Specimen access was provided through the BC Bone and Soft Tissue Tumour Bank (protocol H08-01717). This work was supported by grants from the Canadian Cancer Society Research Institute (Grant \#701582), the Japan Society for the Promotion of Science KAKENHI (Grant \#25893168, \#26713046), a grant from the Fukuoka Foundation for Sound Health and an International Collaborative Grant from the Liddy Shriver Sarcoma Initiative.

\section{Disclosure/conflict of interest}

The authors declare no conflict of interest. 


\section{References}

1 Chen YT, Scanlan MJ, Sahin U, et al. A testicular antigen aberrantly expressed in human cancers detected by autologous antibody screening. Proc Natl Acad Sci U S A 1997;94:1914-1918.

2 Jungbluth AA, Chen YT, Stockert E, et al. Immunohistochemical analysis of NY-ESO-1 antigen expression in normal and malignant human tissues. Int J Cancer 2001;92:856-860.

3 Scanlan MJ, Gure AO, Jungbluth AA, Old LJ, Chen YT. Cancer/testis antigens: an expanding family of targets for cancer immunotherapy. Immunol Rev 2002;188:22-32.

4 Nicholaou T, Ebert L, Davis ID, et al. Directions in the immune targeting of cancer: lessons learned from the cancer-testis Ag NY-ESO-1. Immunol Cell Biol 2006; 84:303-317.

5 Robbins PF, Morgan RA, Feldman SA, et al. Tumor regression in patients with metastatic synovial cell sarcoma and melanoma using genetically engineered lymphocytes reactive with NY-ESO-1. J Clin Oncol 2011;29:917-924.

6 Pollack SM, Jungbluth AA, Hoch BL, et al. NY-ESO-1 is a ubiquitous immunotherapeutic target antigen for patients with myxoid/round cell liposarcoma. Cancer 2012;118:4564-4570.

7 Lai JP, Robbins PF, Raffeld M, et al. NY-ESO-1 expression in synovial sarcoma and other mesenchymal tumors: significance for NY-ESO-1-based targeted therapy and differential diagnosis. Mod Pathol 2012;25:854-858.

8 Hemminger JA, Ewart TA, Scharschmidt TJ, et al. The cancer-testis antigen NY-ESO-1 is highly expressed in myxoid and round cell subset of liposarcomas. Mod Pathol 2013;26:282-288.

9 Hemminger JA, Iwenofu OH. NY-ESO-1 is a sensitive and specific immunohistochemical marker for myxoid and round cell liposarcomas among related mesenchymal myxoid neoplasms. Mod Pathol 2013;26:1204-1210.

10 Nielsen TO, Hsu FD, O’Connell JX, et al. Tissue microarray validation of epidermal growth factor receptor and SALL2 in synovial sarcoma with comparison to tumors of similar histology. Am J Pathol 2003;163:1449-1456.

11 Ng TL, Gown AM, Barry TS, et al. Nuclear beta-catenin in mesenchymal tumors. Mod Pathol 2005;18:68-74.

12 Cheng H, Dodge J, Mehl E, et al. Validation of immature adipogenic status and identification of prognostic biomarkers in myxoid liposarcoma using tissue microarrays. Hum Pathol 2009;40:1244-1251.

13 Steigen SE, Straume B, Turbin D, et al. Clinicopathologic factors and nuclear morphometry as independent prognosticators in KIT-positive gastrointestinal stromal tumors. J Histochem Cytochem 2008;56:139-145.

14 Pacheco M, Nielsen TO. Histone deacetylase 1 and 2 in mesenchymal tumors. Mod Pathol 2012;25:222-230.

15 Endo M, Su L, Nielsen TO. Activating transcription factor 2 in mesenchymal tumors. Hum Pathol 2014;45: 276-284.

16 Terry J, Saito T, Subramanian S, et al. TLE1 as a diagnostic immunohistochemical marker for synovial sarcoma emerging from gene expression profiling studies. Am J Surg Pathol 2007;31:240-246.

17 Demicco EG, Torres KE, Ghadimi MP, et al. Involvement of the PI3K/Akt pathway in myxoid/round cell liposarcoma. Mod Pathol 2012;25:212-221.
18 Hoffman A, Ghadimi MP, Demicco EG, et al. Localized and metastatic myxoid/round cell liposarcoma: clinical and molecular observations. Cancer 2013;119: 1868-1877.

19 Waaijer CJ, de Andrea CE, Hamilton A, van Oosterwijk JG, Stringer SE, Bovee JV. Cartilage tumour progression is characterized by an increased expression of heparan sulphate 6O-sulphation-modifying enzymes. Virchows Arch 2012;461:475-481.

20 Meijer D, Gelderblom H, Karperien M, Cleton-Jansen AM, Hogendoorn PC, Bovee JV. Expression of aromatase and estrogen receptor alpha in chondrosarcoma, but no beneficial effect of inhibiting estrogen signaling both in vitro and in vivo. Clin Sarcoma Res 2011;1:5.

21 Mohseny AB, Szuhai K, Romeo S, et al. Osteosarcoma originates from mesenchymal stem cells in consequence of aneuploidization and genomic loss of Cdkn2. J Pathol 2009;219:294-305.

22 de Graaff MA, Cleton-Jansen AM, Szuhai K, Bovee JV. Mediator complex subunit 12 exon 2 mutation analysis in different subtypes of smooth muscle tumors confirms genetic heterogeneity. Hum Pathol 2013;44: 1597-1604.

23 Willems SM, Schrage YM, Baelde JJ, et al. Myxoid tumours of soft tissue: the so-called myxoid extracellular matrix is heterogeneous in composition. Histopathology 2008;52:465-474.

24 Sun Y, Turbin DA, Ling K, et al. Type I gamma phosphatidylinositol phosphate kinase modulates invasion and proliferation and its expression correlates with poor prognosis in breast cancer. Breast Cancer Res 2010;12:R6.

25 Crozat A, Aman P, Mandahl N, Ron D. Fusion of CHOP to a novel RNA-binding protein in human myxoid liposarcoma. Nature 1993;363:640-644.

26 Powers MP, Wang WL, Hernandez VS, et al. Detection of myxoid liposarcoma-associated FUS-DDIT3 rearrangement variants including a newly identified breakpoint using an optimized RT-PCR assay. Mod Pathol 2010;23:1307-1315.

27 Kilpatrick SE, Doyon J, Choong PF, Sim FH, Nascimento AG. The clinicopathologic spectrum of myxoid and round cell liposarcoma. A study of 95 cases. Cancer 1996;77:1450-1458.

28 Fletcher CDM, Bridge JA, Hogendoorn PCW, Mertens F. World Health Organisation Classification of Tumours. Pathology and Genetics of Tumours of Soft Tissue and Bone, 4th ed. IARC Press: Lyon, 2013.

29 Clark J, Rocques PJ, Crew AJ, et al. Identification of novel genes, SYT and SSX, involved in the $t(X ; 18)$ (p11.2;q11.2) translocation found in human synovial sarcoma. Nat Genet 1994;7:502-508.

30 Ferrari A, Gronchi A, Casanova M, et al. Synovial sarcoma: a retrospective analysis of 271 patients of all ages treated at a single institution. Cancer 2004;101: $627-634$

31 Jungbluth AA, Antonescu CR, Busam KJ, et al. Monophasic and biphasic synovial sarcomas abundantly express cancer/testis antigen NY-ESO-1 but not MAGEA1 or CT7. Int J Cancer 2001;94:252-256.

32 Pollack SM, Li Y, Blaisdell MJ, et al. NYESO-1/LAGE1s and PRAME are targets for antigen specific T cells in chondrosarcoma following treatment with 5-Aza-2deoxycitabine. PLoS One 2012;7:e32165. 